\title{
Daytime noninvasive ventilatory support for patients with ventilatory pump failure: a narrative review
}

\author{
Paolo Banfi $i^{*}$ (D), Paola Pierucci ${ }^{2}$, Eleonora Volpato ${ }^{1,3}$, Antonello Nicolini ${ }^{4}$, Agata Lax', Dominique Robert ${ }^{5,6}$ and \\ John Bach $^{7}$
}

\begin{abstract}
Over the past three decades, the use of noninvasive ventilation or "NIV" to assuage symptoms of hypoventilation for patients with early onset or mild ventilatory pump failure has been extended to up to the use of continuous noninvasive ventilatory support (CNVS) at full ventilatory support settings as a definitive alternative to tracheostomy mechanical ventilation. NVS, along with mechanical insufflation-exsufflation, now provides a noninvasive option for the management of both chronic and acute respiratory failure for these patients. The most common diagnoses for which these methods are useful include chest wall deformities, neuromuscular diseases, morbid obesity, high level spinal cord injury and idiopathic, primary or secondary disorders of the ventilatory control. Thus, NVS is being used in diverse settings: critical care units, medical wards, at home, and in extended care. The aim of this review is to examine the techniques used for daytime support.
\end{abstract}

Keywords: Neuromuscular disorders, Respiratory failure, Noninvasive ventilation/ventilatory support, Mouthpiece ventilation, Intermittent abdominal pressure ventilation

\section{Introduction}

Respiratory muscle insufficiency/failure is defined by the inability to maintain adequate blood oxygenation and carbon dioxide removal $[1,2]$. The underlying pathophysiologic processes causing it are quite different from those typically treated by physicians. Instead of lung/airways disorders that result primarily in oxygenation impairment and air trapping, for patients with ventilatory pump failure hypoxia is secondary to inspiratory and/or expiratory muscle impairment that results in hypoventilation and airway mucus congestion [3, 4]. When patients' respiratory muscles cannot sustain autonomous respiration, the use of mechanical ventilation has become [5] the cornerstone of modern pulmonary medicine and critical care.

Non-invasive ventilation or "NIV" has become synonymous with continuous positive airway pressure and low span bi-level positive airway pressure to treat sleepdisordered breathing (central and obstructive apneas) and acute respiratory failure for patients with lung

\footnotetext{
* Correspondence: pabanfi@dongnocchi.it

${ }^{1}$ IRCCS Fondazione Don Carlo Gnocchi, via Capecelatro, 6620148 Milan, Italy Full list of author information is available at the end of the article
}

disease [5-11]. Observational and randomized controlled studies now strongly support NIV for patients with mild neuromuscular disease (NMD) who are not completely respirator dependent [12-19]. Furthermore, when considering NIV, for patients with lung diseases, airway clearance therapy is addressed [20-22]. Standardized guidelines have been accepted worldwide for the initiation of nocturnal NIV [23-32]. However, for patients with ventilatory pump failure advancing disease can result in patients extending sleep NIV into daytime hours and becoming hypercapnic because of the inadequacy of the pressure support settings. These patients can also require mechanical in-exsufflation to increase cough flows [20-22]. The paucity of literature focused on this topic and failure to recognize the deficiencies in the administration of NIV motivated this review, which aims to highlight means for providing up to continuous noninvasive ventilatory support (CNVS).

There is NVS and invasive mechanical ventilation whether via translaryngeal or tracheostomy tube (TMV) [5-8, 33-36]. However, $80 \%$ of mortality in some diagnoses of patients using tracheostomy ventilation has 
been reported to be due to the tube itself, rather than directly to the disease [37]. On the other hand, while tracheostomy ventilation is associated with increased mortality and diminished quality of life [38], CNVS is permitting patients with no autonomous breathing ability and little to no vital capacity (VC) with diagnoses including spinal muscular atrophy type 1 more than 2 decades [39], post-polio survivors to survive for over 65 years [40], Duchenne muscular dystrophy to survive into their 50s on CNVS for over 25 years [41], etc.. Patients who require daytime NVS can eventually require it around-the-clock with little to no autonomous ability to breathe. For this, full respiratory support settings are necessary and "NIV" or typical bi-level positive airway pressure is inadequate. Thus, CNVS refers to the administration of full ventilatory support settings day and night $[9,13]$. Its benefits include improving gas exchange, symptoms [42], quality of life [43-45], decreasing the incidence of pneumonia [46], facilitating ventilator weaning, resting respiratory muscles, and supporting life for people indefinitely without need to resort to tracheotomy [46]. Tracheostomy tubes, on the other hand, tend to increase ventilator dependence, reduce quality of life, and are associated with reactive depression [47-50].

A literature review and manual selection using PubMed/Medline was undertaken through May 2019. Search terms used were noninvasive ventilation, noninvasive ventilatory support, home ventilation, diurnal or daytime non-invasive ventilation and neuromuscular. Retrospective, prospective, controlled, and noncontrolled studies were included. Abstracts or clinical cases were excluded.

\section{Introduction of daytime support}

The VC is commonly used to assess respiratory muscle weakness. A VC less than 50\% [8] of predicted normal or forced expiratory volume in $1 \mathrm{~s}$ less than $40-50 \%$ predicted along with clear symptoms of sleep hypoventilation, indicate need to introduce sleep NVS. Maximal inspiratory pressure or sniff nasal pressure less than $30-40 \mathrm{~cm} \mathrm{H}_{2} \mathrm{O}$ can also be helpful [21-29]. A decrease in $\mathrm{VC}$ of $25 \%$ or more when going from sitting to supine indicates diaphragm weakness and explains respiratory orthopnea and need for sleep NVS [51]. Typical symptoms of hypoventilation include fatigue, dyspnea, morning headaches, and hypersomnolence. Corroborating signs can include $\mathrm{PaCO}_{2}$ greater than 45 $\mathrm{mmHg}$, nocturnal oximetry saturation $<88 \%$ for 5 consecutive minutes [21], and end-tidal $\mathrm{CO} 2>47 \mathrm{mmHg}$ [52]. Thus, the indication to initiate sleep NVS is symptomatic hypoventilation in the presence of any indications of respiratory muscle dysfunction. Typically, with advancing disease and decreasing VC, sleep NVS users become dyspneic when NVS is discontinued in the morning and nasal NVS is extended NVS into daytime hours [45, 52-60]. While sleep NVS initially normalizes daytime blood gases, further muscle deterioration causes increasing daytime hypercapnia such that when $\mathrm{O}_{2}$ desaturation occurs below 95\% dyspnea results in ever increasing use of NVS throughout daytime hours [57-61].

\section{Daytime support methods \\ Mouthpiece and nasal NVS}

Mouthpiece NVS was first described for CNVS in 1953 to permit iron lung users, with little to no autonomous ability to breathe, to leave iron lungs during the day. Subsequently they often began to refuse to return to iron lungs for sleep so they used mouthpiece NVS for CNVS $[62,63]$. Their ventilator settings were typically pressure control at 18 to, at times for obese patients, 35 or more $\mathrm{cm} \mathrm{H} 2 \mathrm{O}$ for daytime as well as sleep NVS. When volume cycling portable ventilators came onto the market in 1976, CNVS was typically delivered at preset volumes of 800 to $1500 \mathrm{ml}$. The patients took as much of the air as desired for each breath. Besides normalizing $\mathrm{PaO}_{2}, \mathrm{PaCO}_{2}$, and sleep quality, CNVS indisputably prolongs survival for patients with little or no autonomous ability to breathe, 257 such patients were described in 1993 [64].

Mouthpiece NVS is provided via 15 or $22 \mathrm{~mm}$ angled, or straw-type, mouthpieces accessible to the mouth by being supported by a flexible support arm such that the patient can access it with the lips as needed (Fig. 1). Patients can trigger the breaths by creating a small negative pressure (sip and breathe) or placing the mouth on the mouthpiece (Kiss Trigger, Trilogy Ventilator, Respironics Inc., Murrysville Pa) [61]. Any mode can be used but volume preset ventilation with an active circuit permits active lung volume recruitment ("air-stacking"). This is the glottis retention of consecutively delivered volumes of air until a deep lung volume is attained. This maintains pulmonary compliance, increases cough flows and speech volume. A problem can be false alarms when using open circuits [61]. Several new portable ventilators have now a mouthpiece ventilation mode ("MPV mode") that minimizes false alarms [65-68]. Although mouthpiece NVS facilitates speaking, eating, swallowing, and coughing, the patient can discontinue it as desired. There is no dyspnoea or transient oxyhemoglobin desaturations between grabbing the mouth piece for supported breaths when NVS settings are used [68]. For example, if normal minute ventilation is $6000 \mathrm{ml}$, this can be taken in by 12 breaths of $500 \mathrm{ml}$ or 4 of $1500 \mathrm{ml}$. The latter affords more than $10 \mathrm{~s}$ for chewing and swallowing.

The $15 \mathrm{~mm}$ angled mouthpiece and straw-type mouthpiece are easy to grip $[65,68]$. Khirani et al. [67] evaluated 


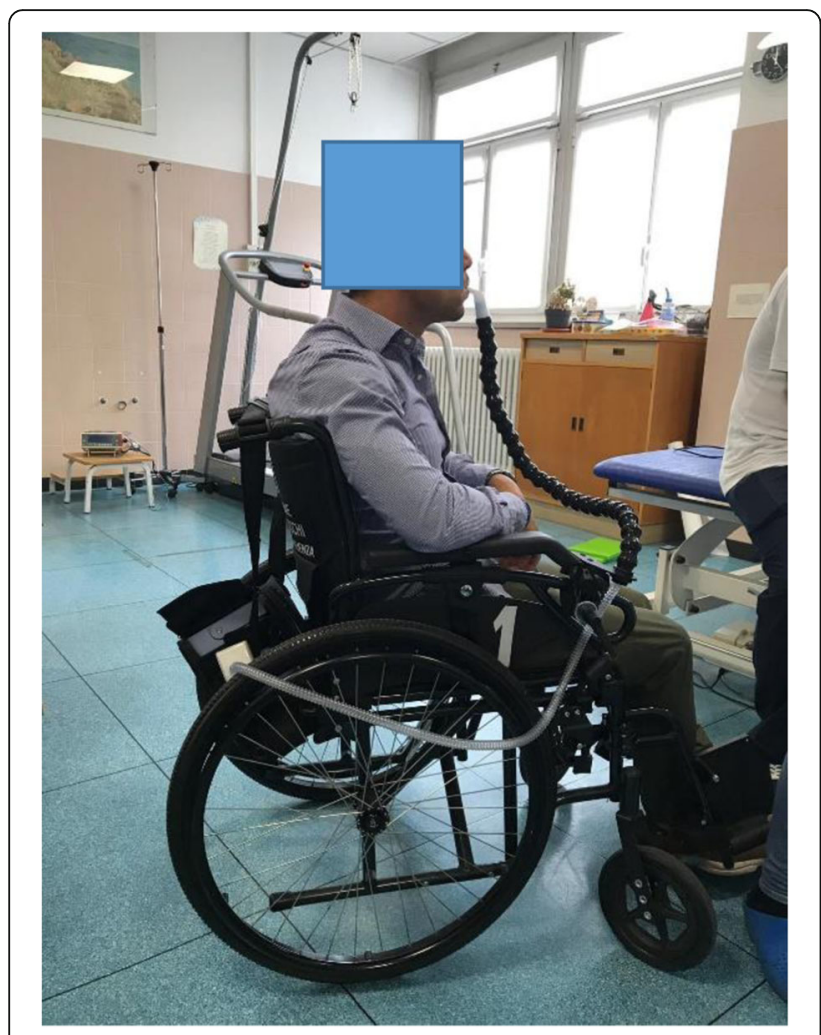

Fig. 1 Mouthpiece ventilation

209 patients, 30 using MPV modes, by questionnaire. These patients associated mouthpiece NVS with less dyspnoea and fatigue and greater facility in speaking and eating. In another study, Nicolini et al. [68] reported an improvement in clinical symptoms, blood gases, and in nocturnal ventilation, sleep related parameters and Health Related Quality of Live scores for kyphoscoliotic patients. While frequent or prolonged disconnections inducing apnoea/hypopnea or hypoventilation have been noted [63, 68], this only occurs when less than NVS settings are used.

Assist control pressure or volume preset ventilation modes with minimal back-up rates and extreme alarm settings are used to avoid nuisance alarms. The Kiss Trigger $^{\mathrm{rm}}$ of the Trilogy $^{\mathrm{Tm}}$ ventilator permits 0 back-up rates [69].

While in the 1950s and 60s $15 \mathrm{~mm}$ angled mouthpiece NVS was used around-the-clock, once a lip cover phalange came onto the market in 1964 this secured the mouthpiece and permitted the delivery of air with little to no mouth leak during sleep (Fig. 1). This was the principal method of sleep NVS until Bach, Alba, Mosher, and Delaubier described nasal NVS in 1987. Today, 3 out of 4 patients prefer nasal over oral interfaces for sleep NVS [70, 71]. Recently, over 700 CNVS dependent muscular dystrophy and amyotrophic lateral sclerosis patients were reported using daytime mouthpiece NVS [72]. However, for those for whom lips are too weak or neck movement inadequate to grab a mouthpiece, daytime nasal NVS has always been preferred over resort to tracheotomy [71].

Skin breakdowns typically only occur when a single nasal interface is used around-the-clock [73, 74]. Daytime NVS can cause airway and mouth dryness that can negatively impact on quality of life and cause airway congestion and possibly inadequate gas-exchange and atelectasis [53]. With typical daytime mouthpiece NVS, however, there is no interface intolerance, difficulty speaking, expectorating or eating, facial deformities, or claustrophobia [16]. While skin discomfort, eye irritation, and other difficulties have been associated with the use of nasal and oronasal interfaces for sleep, none of this is relevant for daytime mouthpiece NVS users or nasal NVS users who use nasal prongs for daytime NVS and daytime mouthpiece or nasal prong NVS users.

\section{Interfaces}

Currently, there are many interfaces commercially available $[74,75]$. They come in a variety of styles and sized i.e. nasal, oro-nasal, full-face, mouthpiece or orophylange. Each has advantages and disadvantages.

- Mouthpiece: Straw-type or $15 \mathrm{~mm}$ angled mouthpieces are grabbed with the lips and teeth [76]. No pressure over the bridge of the nose or need for headgear. It permits use on demand and active lung volume recruitment (air stacking). There are no downsides for daytime use other than mouth and airway dryness. The most commonly used mouthpiece is the $15 \mathrm{~mm}$ angled mouthpiece from Respironics Inc. (Murrysville, Pa) [16, 61, 76].

- .Nasal interface (mask): Although triangular nasal interfaces that cover only the nose are used for sleep, nasal prongs inserted into the nostrils, pillows, nose tip covering interfaces are greatly preferred for daytime support although they may cause nostril dryness. These avoid claustrophobia, permit eating and speech and mouth leaks are not a problem during daytime use. Triangular interfaces cause nasal bridge pressure.

- Oronasal interface (mask): These cover both nose and mouth. Mouth and nasal leaks are greatly reduced. They requires less patient cooperation but interfere with speech, eating, and expectoration. They increase risk of rebreathing and cannot be used during meals.

- Full-face interface (mask): These cover mouth, nose, and eyes. They can be useful for sleep but expose patients to eye redness and dryness; they interfere with speech, eating, and expectoration and can not be used for ongoing daytime NVS [75, 77, 78]. 
- Helmet: A transparent air-tight helmet rather than orofacial interface. These permit speech but the increased dead space and possibility of axillary skin injury prevent its ongoing daytime use $[74,75]$.

\section{Body ventilators}

\section{The intermittent abdominal pressure ventilator}

In 1938 the intermittent abdominal pressure ventilation, or "Pneumobelt, was described by C. J. McSweeney as the Bragg-Paul Pulsator. It was used by 34 patients with acute diphtheritic respiratory muscle paralysis. In the 1940 s, it was improved by Dr. Alvin Barach and his engineer William Smith. After 1946, it became widely used for daytime non-invasive ventilatory support [79-84]. It consists of a corset with an elastic inflatable bladder that fits over the abdomen. The bladder is connected by a hose to a ventilator that deliver up to $2.5 \mathrm{l}$ of air to the bladder and, thereby, to the abdominal wall [82, 83]. This elevates the diaphragm to cause expiration below the functional residual capacity. When the bladder deflates, the diaphragm descends by gravity and inspiration occurs passively. Typically, 300 to $1,000 \mathrm{ml}$ of air are delivered [81]. It went off the market as tracheotomies became the convention but is back on the market today (Dima Inc., Italy). New models avoid clothing catching on the corset buckles and are more comfortable. They are now lightweight, comfortable, easy to done and fit and employ velcro for fastening (Fig. 2) [55]. Nevertheless, mouthpiece NVS users generally prefer mouthpiece NVS because of the greater inspiratory volumes that can be taken in.

Limitations include the fact that its efficiency depends upon the surface area of the chest and the abdomen covered. Since the increase of lung volume is generated by gravity, it is effective only in the sitting position [82], or at least at an angle of $30^{\circ}$ or greater [44]. It is usually not sufficiently effective for very obese or severely scoliotic patients [79]. The current device on the market allows

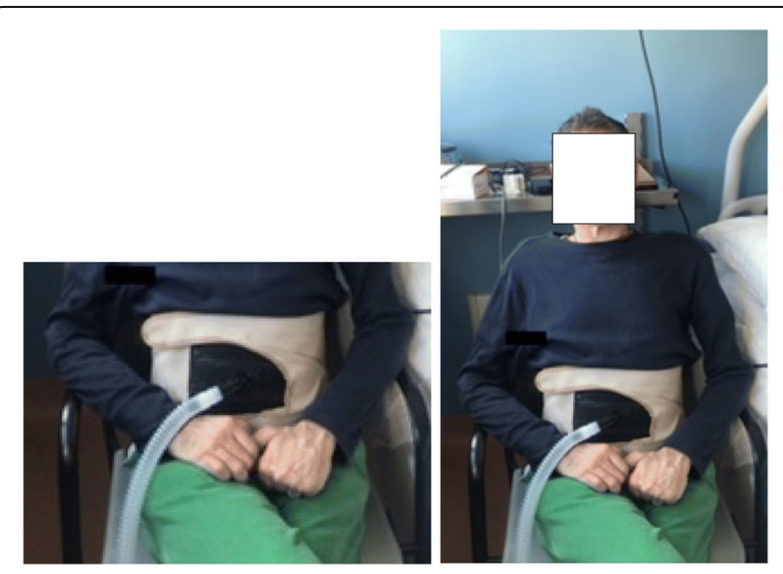

Fig. 2 Intermittent abdominal pressure ventilation by PBelt the user to set the following parameters: - PBelt (pressure inside the PBAir ${ }^{\circ}$ ); -Tinsp (real inspiratory time when the diaphragm descends); - Frequency (respiratory rate); and -Rise Time (time to reach the PBelt). Thus, "Pneumobelt" use is inconspicuous, comfortable, simple to do and use and leaves the face/mouth/nose, and neck free. It is portable and easily installed onto a wheelchair. It facilitates speech, belching to pass flatus and abdominal compression to increase cough flows to help clear secretions. Patients learn to close the glottis just prior to an abdominal compression to increase cough flows. It is also useful for constipation [82]. It also leaves the field of vision free and allows the patient a more normal sense of smell by eliminating facial and invasive interfaces that may be colonized by pathogenic bacteria. Thus, it favors oral nutrition and digestion [82]. In rare cases it is used for sleep with patients sitting [83]. Disadvantages can be food regurgitation during meals (rarely), catching clothing on straps and Velcro closures, redness of the bony prominences, and impossibility to shower or to bath when using it [83, 84]. Furthermore, regular follow up is important because it can become less effective with time $[83,84]$. Its use has also recently been reported for patients with cervical myelopathies [55]. For all these reasons, the "Pneumobelt" is again becoming an important alternative for the patients requiring daytime support.

\section{Negative-pressure ventilation}

Negative pressure ventilation was widely used for both sleep and daytime ventilatory support as iron lungs, pancho, pneumosuit, and pneumowrap ventilators before the advent of NVS in the 1950s. The chest cuirass is a rigid shell fitting over the anterior portion of the chest or over the chest and abdomen. The pneumowrap, poncho, pneumosuit ventilators consisted of a parka suspended over a rigid plastic or metal chest piece. The cuirass and wrapstyle interfaces are powered by large negative pressure ventilators. While mouthpiece NVS facilitates speech, eating, maximizes coughing, and raising one's voice, negative pressure body ventilators don't.

Only the cuirass or chest shell ventilator can be used for daytime support for patients in wheelchairs [8-88]. The ventilator assumes part of the patient's work of breathing and therefore respiratory muscles can rest but not so much as using NVS [89]. During sleep all negative pressure body ventilators cause severe obstructive apneas [90,91].

The biphasic chest shell ventilator (Hayek Inc., England) can certainly provide daytime ventilatory assistance for patients without severe scoliosis, however it is unsightly, restrictive on mobility, and no more effective than "pneumobelts" at providing large tidal volumes for daytime ventilatory support. It has never been preferable to "pneumobelt" or mouthpiece NVS [82]. 


\section{Glossopharyngeal breathing (GPB)}

Patients with at least some bulbar muscle function can often learn to glossopharyngeal breath for ventilator free breathing as well as for "auto" air stacking. This involves the tongue pistoning boluses of air past the glottis and into the lungs. It can be done with the mouth open or, in some cases, via the nose with the mouth closed [92-95]. It can improve speech volume and cough efficacy [95]. The technique can allow increased periods off of NVS or even all day autonomous ability for adept patients despite having little to no VC [96-99].

\section{Mechanical insufflation-exsufflation}

Unfortunately, with the paradigm shift to invasive ventilatory support in the late $1960 \mathrm{~s}$, the Cof-flator, the first mechanical in-exsufflation device, went off the market as did the "Pneumobelt" 15 years later. There had been no major publications on it until 1991. However, like the "Pneumobelt", mechanical in-exsufflation continues to be a practical, convenient, comfortable, and effective method to greatly increase cough flows to expel airway secretions and prevent pneumonia and respiratory failure and to permit extubation of ventilator unweanable patients to CNVS so that tracheotomy could be avoided indefinitely. Neither NIV and NVS should ever be considered in without considering mechanical inexsufflation. In fact, publications reporting that NIV fails and tracheotomy is needed are simply failures to use NVS settings and, most importantly, failures to use mechanical in-exsufflation at the effective settings of 40 to $70 \mathrm{~cm} \mathrm{H}_{2} \mathrm{O}$ via both noninvasive as well as invasive interfaces [100-102].

\section{Training}

It is recommended that staff applying NVS receive appropriate training. Both specific knowledge and clinical experience are necessary. The training needs to be for health care professionals including physicians, nurses and physio/respiratory therapists, and most importantly, for the patient and family [103]. The time required for education and training in NIV/NVS and mechanical inexsufflation is variable. Some authors have suggested that an initial session of two hours three times a month may provide adequate background [104]. With staff experience, sessions can then be distanced in time. Quantifying the experience and skill of a unit's staff is challenging because individuals differ considerably and personnel changes can have important effects [105]. Optimal noninvasive management for daytime, as well as sleep support, requires all team members to be experienced. They must also be skilled in selecting appropriate devices and interfaces, fitting to optimize comfort, and adjusting the ventilator to efficiently alleviate respiratory distress and provide optimal settings. Nurses and physiotherapists need to be knowledgeable in regards to monitoring all stages of this in order to better train the patient and family.

\section{Conclusions}

Daytime NVS is crucial for patients with advanced ventilatory pump failure to avoid acute on chronic respiratory failure and invasive airway tubes. Precise criteria for daytime NIV/NVS initiation are totally unnecessary since patients extend nocturnal use into daytime hours on their own. Interfaces and access to mechanical inexsufflation play key roles for NVS success. Glossopharyngeal breathing can also be a very effective means of daytime ventilatory support. Body ventilators may become a more prominent feature of daytime support with time. Further research in this emerging field is strongly warranted in order to further improve the quality of breathing and living for these fragile patients.

\section{Abbreviations \\ NIV: Noninvasive Ventilation; CNVS: Continuous Noninvasive Ventilatory Support; NVS: Non-invasive Ventilatory Support; VC: Vital Capacity;}

MPV: Mouthpiece Ventilation

\section{Acknowledgements}

No acknowledgements.

\section{Authors' contributions}

PB and PP conception of the review, revised the literature and drafting the review, EV drafting the review and acquisition of the literature, $A N$ and $A L$ acquisition of the literature according with $P B$ and $P P$, and $D R$ and JB revised article and literature. All authors read and approved the final manuscript.

\section{Funding}

The authors declare no funding for this review.

Availability of data and materials

Not available.

Ethics approval and consent to participate

Normally not available for review.

Consent for publication

We have the patient's signed consent for the publication of the pictures.

Competing interests

The authors declare that they have no competing interest.

\section{Author details}

${ }^{1}$ IRCCS Fondazione Don Carlo Gnocchi, via Capecelatro, 6620148 Milan, Italy. ${ }^{2}$ Cardio Thoracic Department, Respiratory and Sleep Disorders Unit, Bari Policlinic, Bari, Italy. ${ }^{3}$ Department of Psychology, Università Cattolica del Sacro Cuore, Milan, Italy. ${ }^{4}$ Respiratory Rehabilitation Unit, ASL 4 Chiavarese, Hospital of Sestri Levante, Sestri Levante, Italy. ${ }^{5}$ Hospices Civils de Lyon, Hôpital Edouard Herriot, Service de Réanimation Médicale, Lyon, France. ${ }^{6}$ Université de Lyon, Université Claude Bernard Lyon 1, Lyon, France. 'Department of Physical Medicine and Rehabilitation, Rutgers University New Jersey Medical School, Newark, USA.

Received: 25 July 2019 Accepted: 31 October 2019

Published online: 30 November 2019

\section{References}

1. Roussos C, Koutsoukou A. Respiratory failure. Eur Respir J. 2003;47:3s-14s.

2. Friedman ML, Nitu ME. Acute respiratory failure in children. Pediatr Ann. 2018;47(7):e268-73. 
3. Bach JR. Pathophysiology of paralytic/restrictive pulmonary syndromes. In: Bach JR, editor. Pulmonary rehabilitation: the obstructive and paralytic conditions. Philadelphia: Hanley \& Belfus; 1996. p. 275-83.

4. Bach JR. Physiology and pathophysiology of hypoventilation: ventilatory vs. oxygenation impairment. In: Bach JR, editor. Noninvasive mechanical ventilation. Philadelphia: Hanley \& Belfus; 2002. p. 25-45.

5. Carlucci A, Richard JC, Wysocki M, Lepage E, Brochard L. SRLF collaborative group on mechanical ventilation. Noninvasive versus conventional mechanical ventilation. An epidemiologic survey. Am J Respir Crit Care Med. 2001;163:874-80.

6. Mas A, Masip J. Noninvasive ventilation in acute respiratory failure. Int J Chron Obstruct Pulmon Dis. 2014;9:837-52. https://doi.org/10.2147/COPD. S42664 eCollection 2014.

7. Demoule A, Girou E, Richard JC, Taillé S, Brochard L. Increased use of noninvasive ventilation in French intensive care units. Intensive Care Med. 2006;32:1747-55.

8. Ward S, Chatwin M, Heather S, Simonds AK. Randomised controlled trial of non-invasive ventilation (NIV). Thorax. 2005;60:1019-24.

9. Crescimanno G, Greco F, D'Alia R, Messina L, Marrone O. Quality of life in long term ventilated adult patients with Duchenne muscular dystrophy. Neuromuscul Disord. 2019:569-75

10. Crimi C, Pierucci P, Carlucci A, Cortegiani A, Gregoretti C. Long -term ventilation in neuromuscular patients: review of concerns, beliefs, and ethical dilemmas. Respiration. 2019;97(3):185-96.

11. Perrin C, Rolland F, Berthier F, Duval Y, Jullien V. Noninvasive ventilation for acute respiratory failure in a pulmonary department. Rev Mal Respir. 2015; 32(9):895-902.

12. Bello G, De Pascale G, Antonelli M. Noninvasive ventilation. Clin Chest Med. 2016;37(4):711-21. https://doi.org/10.1016/j.ccm.2016.07.011.

13. Gonzalez J, Sharshar T, Hart N, Chadda K, Raphaël JC, Lofaso F. Air leaks during mechanical ventilation as a cause of persistent hypercapnia in neuromuscular disorders. Intensive Care Med. 2003; 29(4):596-602.

14. Sangeeta Mehta, And Nicholas S. Hill. Noninvasive Ventilation. Am J Respir Crit Care Med. 2001;163:2.

15. Hess DR. Noninvasive ventilation for neuromuscular disease. Clin Chest Med. 2018;39:437-47.

16. Toussaint M, Steens M, Wasteels $G$, Soudon P. Diurnal ventilation via mouthpiece: survival in end-stage Duchenne patients. Eur Respir J. 2006;28:549-55.

17. Radunovic A, Annane D, Rafiq MK, Brassington R, Mustfa N. Mechanical ventilation for amyotrophic lateral sclerosis/motor neuron disease. Cochrane Database Syst Rev. 2017;10:CD004427. https://doi.org/10.1002/14651858. CD004427.pub4.

18. Bach JR, Takyi SL. Physical medicine interventions to avoid acute respiratory failure and invasive airway tubes. PM R. 2015;7:871-7.

19. Gomez-Merino E, Bach JR. Duchenne muscular dystrophy: prolongation of life by noninvasive ventilation and mechanically assisted coughing. Am J Phys Med Rehabil. 2002;81:411-5.

20. Vianello A, Corrado A, Arcaro G, Gallan F, Ori C, Minuzzo M, Bevilacqua M. Mechanical insufflation-exsufflation improves outcomes for neuromuscular disease patients with respiratory tract infections. Am J Phys Med Rehabil. 2005;84:83-8.

21. Morelot-Panzini C, Bruneteau G, Gonzalez-Bermejo J. NIV in amyotrophic lateral sclerosis: the 'when' and 'how' of the matter. Respirology. 2019;24(6):521-30.

22. Khamankar N, Coan G, Weaver B, Mitchell CS. Associative increases in amyotrophic lateral sclerosis survival duration with non-invasive ventilation initiation and usage protocols. Front Neurol. 2018;9:578.

23. Simonds AK. Recent advances in respiratory care for neuromuscular disease. Chest. 2006;130(6):1879-86

24. Sheers N, Howard ME, Berlowitz DJ. Respiratory adjuncts to NIV in neuromuscular disease. Respirology. 2019;24(6):512-20.

25. Shneerson JM, Simonds AK. Noninvasive ventilation for chest wall and neuromuscular disorders. Eur Respir J. 2002;20(2):480-7.

26. Hess DR. The growing role of noninvasive ventilation in patients requiring prolonged mechanical ventilation. Respir Care. 2012;57:900-18.

27. Finder DJ, Binkrant D, Carl J, et al. Respiratory care of the patient with Duchenne muscular dystrophy: ATS consensus statement. Am J Respir Crit Care Med. 2004;170:456-65.

28. Birnkrant DJ, Bushby K, Bann CM, Apkon SD, Blackwell A, Colvin MK, Care Considerations Working Group. Diagnosis and management of Duchenne muscular dystrophy, part 3: Primary care, emergency management, psychosocial care, and transitions of care across the lifespan. Lancet Neurol. 2018;17(5):445-55.

29. Bach JR. Noninvasive respiratory management of patients with neuromuscular disease. Ann Rehabil Med. 2017:41:519-36.

30. Ramirez A, Delord V, Khirani S, Leroux K, Cassier S, Kadlub N, et al. Interfaces for long-term noninvasive positive pressure ventilation in children. Intensive Care Med. 2012;38(4):655-62.

31. Chatwin M, Tan HL, Bush A, Rosenthal M, Simonds AK. Long term noninvasive ventilation in children: impact on survival and transition to adult care. PLoS One. 2015;1:10.

32. Hamada S, Ishikawa Y, Aoyagi T, Ishikawa Y, Minami R, Bach JR. Indicators for ventilation use in Duchenne muscular dystrophy. Respir Med. 2011:105:625-9.

33. Butz M, Wollinsky KH, Wiedemuth-Catrinescu U, Sperfeld A, Winter $\mathrm{S}$, Mehrkens $\mathrm{HH}$, et al. Longitudinal effects of noninvasive positive-pressure ventilation in patients with amyotrophic lateral sclerosis. Am J Phys Med Rehabil. 2003;82(8):597-604.

34. Hess DR. Non invasive ventilation for acute respiratory failure. Respir Care. 2013:58:950-72

35. Squadrone E, Frigerio P, Fogliati C, Gregoretti C, Conti G, Antonelli M, et al. Noninvasive vs invasive ventilation in COPD patients with severe acute respiratory failure deemed to require ventilatory assistance. Intensive Care Med. 2004;30(7):1303-10.

36. Luo F, Annane D, Orlikowski D, He L, Yang M, Zhou M, et al. Invasive versus non-invasive ventilation for acute respiratory failure in neuromuscular disease and chest wall disorders. Cochrane Database Syst Rev. 2017;12: CD008380.

37. Bach JR. Amyotrophic lateral sclerosis: communication status and survival with ventilatory support. Am J Phys Med Rehabil. 1993;72(6):343-9.

38. Bach JR. A comparison of long-term ventilatory support alternatives from the perspective of the patient and care giver. Chest. 1993;104(6):1702-6.

39. Bach JR. Point: is non-invasive ventilation always the most appropriate manner of long-term ventilation for infants with spinal muscular atrophy type 1? Yes, almost always? Chest. 2016;151(5):962-5.

40. Bach JR. Noninvasive respiratory management of patients with neuromuscular disease. Ann Rehabil Med. 2017:41(4):1-20.

41. Villanova M, Brancalion B, Mehta AD. Duchenne muscular dystrophy: life prolongation by noninvasive ventilatory support. Am J Phys Med Rehabil. 2014;93(7):595-9. https://doi.org/10.1097/PHM.0000000000000074.

42. Toussaint $M$, Chatwin M, Soudon P. Mechanical ventilation in Duchenne patients with chronic respiratory insufficiency: clinical implications of 20 years published experience. Chron Respir Dis. 2007;4:167-77.

43. Bach JR. Noninvasive respiratory Management of Patients with neuromuscular disease. Ann Rehabil Med. 2017;41(4):519-38. https://doi.org/ 10.5535/arm.2017.41.4.519.

44. Hess DR. Noninvasive ventilation in neuromuscular disease: equipment and application. Respir Care. 2006:51(8):896-911 discussion 911-2.

45. Tsolaki V, Pastaka C, Kostikas K, Karetsi E, Dimoulis A, Zikiri A, et al. Noninvasive ventilation in chronic respiratory failure: effects on quality of life. Respiration. 2011;81:402-10.

46. Bach JR, Martinez D. Duchenne muscular dystrophy: prolongation of survival by noninvasive interventions. Respir Care. 2011;56(6):744-50.

47. Pinto T, Chatwin M, Banfi P. Winck JC, Nicolini A. Mouthpiece ventilation and complementary techniques in patients with neuromuscular disease: a brief clinical review and update. Chron Respir Dis. 2017:14(2):187-93.

48. Bach JR, Gonçalves MR, Hamdani I, Winck JC. Extubation of patients with neuromuscular weakness: a new management paradigm. Chest. 2010;137: 1033-9; Prepublished online December 29, 2009. https://doi.org/10.1378/ chest.09-2144.

49. Bach JR, Goncalves MR, Rodriguez PL, Saporito L, Soares L. Cuff deflation: rehabilitation in critical care. Am J Phys Med Rehabil. 2014;93:719-23.

50. Sancho J, Servera E, Díaz JL, Banuls P, Marín J. Home tracheotomy mechanical ventilation in patients with amyotrophic lateral sclerosis: causes, complications and 1-year survival. Thorax. 2011:66:948-52.

51. Caggiano S, Khirani S, Dabaj I, Cavassa E, Amaddeo A, Arroyo JO, et al. Diaphragmatic dysfunction in SEPN1-related myopathy. Neuromuscul Disord. 2017;27(8):747-55. https://doi.org/10.1016/j.nmd.2017.04.010. Epub 2017 Apr 26.

52. Georges M, Golmard JL, Llontop C, Shoukri A, Salachas F, Similowski T, et al. Initiation of non-invasive ventilation in amyotrophic lateral sclerosis and clinical practice guidelines: single-Centre, retrospective, descriptive study in 
a national reference Centre. Amyotroph Lateral Scler Frontotemporal Degener. 2017;18(1-2):46-52.

53. Wood KE, Flaten AL, Backes WJ. Inspissated secretions: a life-threatening complication of prolonged noninvasive ventilation. Respir Care. 2000;45(5): 491-3.

54. Dettenmeier PA, Jackson NC. Chronic hypoventilation syndrome: treatment with non-invasive mechanical ventilation. AACN Clin Issues Crit Care Nurs. 1991;2(3):415-31.

55. Banfi P, Volpato E, Bach JR. Efficacy of new intermittent abdominal pressure ventilator for post-ischemic cervical myelopathy ventilatory insufficiency. Multidiscip Respir Med. 2019;14:4.

56. Bach JR, Radbourne M, Potpally N, Chiou M. A mechanical intermittent abdominal pressure ventilator: a case report. Am J Phys Med Rehabil. 2019. https://doi.org/10.1097/PHM.0000000000001151 [Epub ahead of print].

57. Orlikowski D, Prigent $H$, Salva M-AQ, Heming N, Chaffaut C, Chevret $S$, et al. Prognostic value of nocturnal hypoventilation in neuromuscular patients. Neuromuscul Disord. 2017:27:326-30.

58. Ambrosino N, Confalonieri M, Crescimanno G, Vianello A, Vitacca M. The role of respiratory management of Pompe disease. Respir Med. 2013;107(8): 1124-32.

59. Grigg-Damberger MM, Wagner LK, Brown LK. Sleep hypoventilation in patients with neuromuscular diseases. Sleep Med Clin. 7(2012):667-87.

60. Ogna A, Quera Salva MA, Prigent H, Mroue G, Vaugier I, et al. Nocturnal hypoventilation in neuromuscular disease: prevalence according to different definitions issued from the literature. Sleep Breath. 2016;20(2):575-81.

61. Bedard ME, Mc Kim DA. Daytime mouthpiece for continuous noninvasive ventilation in individuals with amyotrophic lateral sclerosis. Respir Care. 2016:10:1341-8

62. Nicolini A, Parrinello L, Grecchi B, Braido F, Baiardini I, Ghirotti C, et al. Diurnal mouthpiece ventilation and nocturnal non-invasive ventilation versus tracheostomy invasive ventilation in patients with amyotrophic lateral sclerosis. Panminerva Med. 2019. https://doi.org/10.23736/S0031-0808. 19.03644-9. [Epub ahead of print].

63. Fiorentino G, Annunziata A, Gaeta AM, Lanza M, Esquinas A. Continuous noninvasive ventilation for respiratory failure in patients with amyotrophic lateral sclerosis: current perspectives. Degener Neurol Neuromuscul Dis. 2018;8:55-61.

64. Bach JR, Alba AS, Saporito LR. Intermittent positive pressure ventilation via the mouth as an alternative to tracheostomy for 257 ventilator users. Chest. 1993;103(1):174-82.

65. Pinto T, Chatwin M, Banfi P, Winck JC, Nicolini A. Mouthpiece ventilation and complementary techniques in patients with neuromuscular disease: a brief clinical review and update. Chron Resp Dis. 2016.

66. Garuti G, Nicolini A, Grecchi B, Lusuardi M, Winck JC, Bach JR. Open circuit mouthpiece ventilation: concise clinical review. Rev Port Pneumol. 2014;20:211-8.

67. Khirani $S$, et al. Evaluation of ventilators for mouthpiece ventilation in neuromuscular disease. Respir Care. 2014;59:1329-37.

68. Nicolini A, Barlascini C, Piroddi IM, Garuti G, Banfi PI. Effectiveness and safety of mouthpiece ventilation and nocturnal non-invasive ventilation in patients with kyphoscoliosis: Short and long-term outcomes after an episode of acute respiratory failure. Rev Port Pneumol. 2016;22(2):75-81. https://doi.org/ 10.1016/j.rppnen.2015.09.009 Epub 2015 Dec 31.

69. Carlucci A, Mattei A, Rossi V, Paracchini E, Santi Raineri SM, Gregoretti C. Ventilator settings to avoid nuisance alarms during mouthpiece ventilation. Resp Care. 2016;61:462-7.

70. Bach JR, Alba AS, Mosher R, Delaubier A. Intermittent positive pressure ventilation via nasal access in the management of respiratory insufficiency. Chest. 1987:92(7):168-70

71. Bach JR, Alba AS. Management of chronic alveolar hypoventilation by nasal ventilation. Chest. 1990;97(1):52-7.

72. Goncalves MR, Bach JR, Ishikawa Y, Saporito L, Winck JC. Continuous noninvasive ventilatory support outcomes for neuromuscular disease: a multicentre collaboration and literature review. Pulmonol. 2019. https://doi. org/10.1016/j.pulmoe.2019.05.006 [Epub ahead of print].

73. Navalesi P, Fanfulla F, Frigerio P, Gregoretti C, Nava S. Physiologic evaluation of noninvasive mechanical ventilation delivered with three types of masks in patients with chronic hypercapnic respiratory failure. Crit Care Med. 2000; 28(6):1785-90.

74. Nava S, Navalesi P, Gregoretti C. Interfaces and humidification for noninvasive mechanical ventilation. Respir Care. 2009;54(1):71-84.
75. Sferrazza-Papa GF, Di Marco F, Akoumianaki E, Brochard L. Recent advance in interfaces for non invasive ventilation : from bench studies to practical issues. Min Anestesiol. 2012;78:1146-53.

76. Ogna A, Prignent H, Falaize L, Leroux K, Santos D, Vaugier I, et al. Bench evaluation of commercially available and newly developed interfaces for mouthpiece ventilation. Clin Resp J. 2018;12:890-4.

77. Bach J, Sortor SM, Saporito LR. Interfaces for non-invasive intermittent positive pressure ventilatory support in North America. Eur Respir Rev. 1993; 3(12):254-9.

78. Criner GJ, Travaline JM, Brennan KJ, Kreimer DT. Efficacy of a new full face mask for noninvasive positive pressure ventilation. Chest. 1994; 106(4):1109-15.

79. Adamson JP, Lewis L, Stein JD. Application of abdominal pressure for artificial respiration. JAMA. 1959;169:1613-917.

80. Hill NS. Clinical applications of body ventilators. Chest. 1986;90:897-905.

81. Ayoub J, Milane J, Targhetta R, Prioux J, Chamari K, Arbeille P, Jonquet $\mathrm{O}$, Bourgeois JM, Prefaut $\mathrm{C}$. Diaphragm kinetics during pneumatic belt respiratory assistance: a sonographic study in Duchenne muscular dystrophy. Neuromuscul Disord. 2002;12(6):56975.

82. Bach JR, Alba AS. Intermittent abdominal pressure ventilator in a regimen of noninvasive ventilatory support. Chest. 1991;99(3):630-6.

83. Yang GF, Alba A, Lee $M$, Khan A. Pneumobelt for sleep in the ventilator user: clinical experience. Arch Phys Med Rehabil. 1989;70(9):707-11 (confirmed).

84. Miller HJ, Thomas E, Wilmot CB. Pneumobelt use among high quadriplegic population. Arch Phys Med Rehabil. 1988;69(5):369-72 (confirmed).

85. Shneerson JM. Non-invasive and domiciliary ventilation: negative pressure techniques. Thorax. 1991:46:131-5.

86. Bach JR, Gonçalves MR, Hon A, Ishikawa Y, De Vito EL, Prado F, et al. Changing trends in the management of end-stage neuromuscular respiratory muscle failure: recommendations of an international consensus. Am J Phys Med rehabil. 2013;92:267-77.

87. Gilmartin ME. Body ventilators. Equipment and techniques. Respir Care Clin N Am. 1996;2(2):195-222

88. Corrado A, Gorini M. Long-term negative pressure ventilation. Respir Care Clin N Am. 2002;8:545-57.

89. Glérant JC, Jounieaux V, Parreira VF, Dury M, Aubert G, Rodenstein DO. Effects of intermittent negative pressure ventilation on effective ventilation in normal awake subjects. Chest. 2002;122(1):99-107.

90. Sanna A, Veriter C, Stănescu D. Upper airway obstruction induced by negative-pressure ventilation in awake healthy subjects. J Appl Physio (1985). 1993;75(2):546-52.

91. Bach JR, Penek J. Obstructive sleep apnea complicating negative pressure ventilatory support in patients with chronic paralytic/restrictive ventilatory dysfunction. Chest. 1991;99(6):1386-93.

92. Collier CR, Dail CW, Affeldt JE. Mechanics of glossopharyngeal breathing. J Appl Physiol. 1956:8:580-4

93. Maltais F. Glossopharyngeal breathing. Am J Respir Crit Care Med. 2011;184:381.

94. LoMauro A, Banfi P, D'Angelo MG, Aliverti A. Glossopharyngeal breathing can allow a lung expansion greater than inspiratory capacity in muscular dystrophy. Eur Respir J. 2019. https://doi.org/10.1183/13993003.01938-2018.

95. Loring SH, O'Donnell CR, Butler JP, Lindholm P, Jacobson F, Ferrigno M. Transpulmonary pressures and lung mechanics with glossopharyngeal insufflation and exsufflation beyond normal lung volumes in competitive breath-hold divers. J Appl Physiol. 2007;102:841-6. https://doi.org/10.1152/ japplphysiol.00749.2006.

96. Boussuges A, Gavarry O, Bessereau J, Coulange M, Bourc'his M, Rossi P. Glossopharyngeal insufflation and breath-hold diving: the more, the worse? Wilderness Environ Med. 2014;25:466-71. https://doi.org/10.1016/j.wem. 2014.04.010.

97. Bianchi C, Carrara R, Khirani S, Tuccio MC. Independent cough flow augmentation by glossopharyngeal breathing plus table thrust in muscular dystrophy. Am J Phys Med Rehabil. 2014;93:43-8. https://doi.org/10.1097/ PHM.0b013e3182975bfa.

98. Bach JR, Bianchi C, Vidigal-lopes M, Turi S, Felisari G. Lung inflation by glossopharyngeal breathing and "air stacking" in Duchenne muscular dystrophy. Am J Phys Med Rehabil. 2007;86(4):295-300.

99. Bach JR, Alba AS, Bodofsky E, Curran FJ, Schultheiss M. Glossopharyngeal breathing and noninvasive aids in the management of post-polio respiratory insufficiency. Birth Defects Orig Artic Ser. 1987;23(4):99-113. 
100. Bach JR, Sinquee D, Saporito LR, Botticello AL. Efficacy of mechanical insufflation-exsufflation in extubating unweanable subjects with restrictive pulmonary disorders. Respir Care. 2015;60(4):477-83.

101. Bach JR, Gonçalves MR, Hamdani I, Winck JC. Extubation of unweanable patients with neuromuscular weakness: a new management paradigm. Chest. 2010;137(5):1033-9.

102. Hull J, Aniapravan R, Chan E, Chatwin M, Forton J, Gallagher J, et al. British Thoracic Society guideline for respiratory management of children with neuromuscular weakness. Thorax. 2012;67(Suppl 1):i1-i40.

103. Habib K, Burnes KB, Ciobanu L, El-Khabib M, Nicolini A, Vargas N, et al. Non invasive ventilation: education and training: A narrative analysis and international consensus document. Adv Resp Med. 2019; ahead apub.

104. Simonds AK. Noninvasive ventilation support: a practical handbook. London: Arnold; 2001. p. 230-45.

105. Nicolini A, Piroddi IMG, Barlascini C. How to enhance experience and skill of non invasive ventilation? Suggestions from the literature. Shortness Breath. 2015:4:19-25.

\section{Publisher's Note}

Springer Nature remains neutral with regard to jurisdictional claims in published maps and institutional affiliations.

Ready to submit your research? Choose BMC and benefit from:

- fast, convenient online submission

- thorough peer review by experienced researchers in your field

- rapid publication on acceptance

- support for research data, including large and complex data types

- gold Open Access which fosters wider collaboration and increased citations

- maximum visibility for your research: over $100 \mathrm{M}$ website views per year

At $\mathrm{BMC}$, research is always in progress.

Learn more biomedcentral.com/submissions 